Diabetologe 2014 · 10:274-275

DOI 10.1007/s11428-013-1115-2

Online publiziert: 12. Juni 2014

(c) Springer-Verlag Berlin Heidelberg 2014
B. Kulzer · N. Hermanns

Diabetes-Zentrum Mergentheim, Forschungsinstitut Diabetes-Akademie Bad Mergentheim (FIDAM)

\section{Patientenschulung bei Diabetes mellitus}

\section{Licht und Schatten}

Die strukturierte Patientenschulung bei Diabetes hat in Deutschland eine lange Tradition. Besonders durch die Pionierarbeit der Düsseldorfer Arbeitsgruppe um Prof. Michael Berger, wurden in den 1980er Jahren Strukturen geschaffen, die noch heute die Schulungslandschaft prägen.

Mit der Entwicklung der ersten strukturierten Schulungs- und Behandlungsprogramme für Menschen mit Typ-1und Typ-2-Diabetes wurde die Grundlage für die strukturierte Diabetesschulung in Deutschland geschaffen.

Die Evaluation der Schulungsprogramme war ein wesentlicher Grund für die Erstattungsfähigkeit der Schulung, da damit überzeugende Belege für die Wirksamkeit der Schulung vorlagen. Berger und seine Mitarbeiter waren immer der Auffassung, dass die strukturierte Schulung eine unverzichtbare evidenzbasierte Therapiemaßnahme des Diabetes und eine Diabetestherapie ohne ausreichende Schulung einen schwerwiegenden Behandlungsfehler darstellen. Dem ist auch heute nichts hinzuzufügen.

Auch der Begriff „Schulungs-und Behandlungsprogramme" stammt von der Arbeitsgruppe um Prof. Berger und stellt damit sehr deutlich heraus, dass die $\mathrm{Pa}$ tientenschulung im Zusammenhang mit der medizinischen Behandlung erfolgen sollte. Diesem Ansatz folgten die Autoren der 2012 publizierten Nationalen VersorgungsLeitlinie „Strukturierte Schulungsprogramme“ [1], die ebenfalls betonten, dass die strukturierte Schulung ein integraler Bestandteil der Diabetestherapie ist und durch speziell weitergebildete Schulungskräfte in einer ärztlich geleiteten Einrichtung oder in enger Kooperation mit einer ärztlichen Einrichtung, in der die ärztliche Betreuung der Menschen mit Diabetes erfolgt, angeboten werden soll.

Des Weiteren wegweisend war die Initiative von Prof. Berger, sich seitens der Deutschen Diabetes-Gesellschaft (DDG) um eine Qualifizierung der Schulungskräfte zu kümmern. Der erste Diabetesberaterkurs in Düsseldorf war der Ausgangspunkt für eine echte Erfolgsgeschichte: Bis zum 31.12.2013 wurden durch die DDG 3408 Diabetesberater/innen DDG und 7180 Diabetes-Assistenten/innen DDG ausgebildet. Damit gibt es im weltweiten Vergleich in Deutschland am meisten ausgebildete Schulungskräfte für Diabetes.

Aber trotz dieser sehr erfolgreichen Entwicklung der Patientenschulung in Deutschland bestehen noch immer nichtgelöste Probleme, die schwerpunktmäßig in diesem Leitthemenheft behandelt werden. Obwohl es überzeugende Belege für die Wirksamkeit einer strukturierten Schulung von Menschen mit erhöhtem Risiko für die Diabetesentwicklung auf der Basis von Methoden zur Lebensstilintervention gibt, werden bislang solche Programme in Deutschland kaum angewendet und von den Krankenkassen auch nicht gefördert. In dem 1. Beitrag unserer Arbeitsgruppe (Kulzer et al.) werden das Konzept und die wichtigsten Evaluationsergebnisse von „Prävention des Diabetes - Selbst aktiv werden“ (PRAEDIAS), einem deutschen Diabe- tespräventionsprogramm, vorgestellt. Damit liegen auch für den deutschsprachigen Raum belastbare Daten vor, die zeigen, dass strukturelle Maßnahmen zur Verhaltensprävention bei Typ-2-Diabetes effektiv sind. Bislang liegen ebenfalls nur wenige Daten darüber vor, wie die Schulung in der klinischen Praxis angewendet wird. Diese Lücke schließen die beiden Beiträge von Bergis et al. sowie Ehrmann et al., die über die Ergebnisse eine groß angelegten Versorgungsstudie („PRIMASCOPE“) zur Schulungssituation in Schwerpunktpraxen berichten. Die Ergebnisse zeigen, dass beinahe jeder 4. Patient mit Typ-1-Diabetes und jeder 3. mit Typ-2-Diabetes bislang nicht geschult ist. Bemerkenswert ist auch der geringe Kenntnisstand von Diabetologen bezüglich neuer, moderner Schulungsprogramme, obgleich sie diese als qualitativ sehr viel besser einschätzen als die älteren Programme. Maier et al. geben in ihrem Beitrag erstmals einen Überblick über die aktuelle Vertragssituation für Schulungsprogramme in Deutschland.

\section{$>$}

Im Hinblick auf die Umsetzung und Verfügbarkeit von Schulungsprogrammen bestehen in Deutschland noch große Unterschiede.

Es erstaunt, welche große Unterschiede hinsichtlich der Vertragssituation von Schulungsprogrammen bestehen, obgleich alle der aufgelisteten Programme wissenschaftlich evaluiert wurden und vom Bundesversicherungsamt für die Disease-Management-Programme 
(DMP) zugelassen sind. Hier besteht ein deutlicher Bedarf nach Harmonisierung der verschiedenen Vertragsbedingungen. Obwohl die DMP-Anforderungen an die Schulung auch für den stationären Bereich gelten, stellt die immer kürzere durchschnittliche stationäre Verweildauer die stationäre Schulung zunehmend vor Probleme. Wie Prof. Schiel in seinem Beitrag berichtet, hat sich der Ausschuss „Qualität, Schulung und Weiterbildung (QSW)“ der DDG mit dieser Problematik beschäftigt und mit dem Konzept der „modularen Schulung“ eine Kurskorrektur im Hinblick auf die Bewertung der stationären Schulung im Zertifizierungsprozess vorgenommen.

Wir hoffen, dass Ihnen das Lesen dieses Schwerpunkthefts Spaß macht und Sie wichtige Impulse für die Umsetzung sowie Weiterentwicklung der Patientenschulung bekommen.

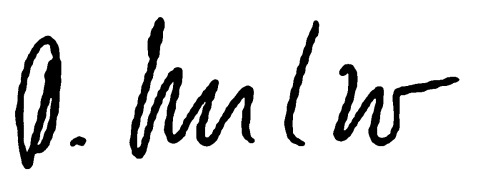

B. Kulzer

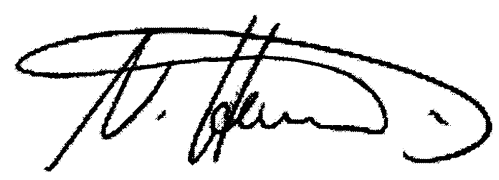

N. Hermanns
Korrespondenzadresse

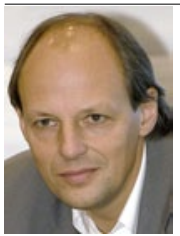

PD Dr. phil. Dipl.-Psych.

\section{B. Kulzer}

Diabetes-Zentrum

Mergentheim,

Forschungsinstitut Diabetes-

Akademie Bad Mergentheim (FIDAM)

Theodor Klotzbücher Str. 12, 97980 Bad Mergentheim Kulzer@diabetes-zentrum.de

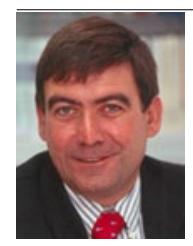

Prof. Dr. N. Hermanns

Diabetes-Zentrum

Mergentheim,

Forschungsinstitut Diabetes-

Akademie Bad Mergentheim

(FIDAM)

Theodor Klotzbücher Str. 12,

97980 Bad Mergentheim

hermanns@

diabetes-zentrum.de

\section{Einhaltung ethischer Richtlinien}

Interessenskonflikt. B. Kulzer und N. Hermanns geben an, dass kein Interessenkonflikt besteht.

\section{Literatur}

1. Bundesärztekammer, Kassenärztliche Bundesvereinigung, Arbeitsgemeinschaft der Wissenschaftlichen Medizinischen Fachgesellschaften (2012) Nationale VersorgungsLeitlinie Diabetes. Strukturierte Schulungsprogramme - Langfassung. Version 1.0. http://www.versorgungsleitlinien.de/themen/ diabetes2/dm2_schulung. Zugegriffen: 25. April 2014

\section{Absterben von Betazellen verhindern}

Wissenschaftler der Universität Bremen haben ein Schlüsselprotein identifiziert, das für das Absterben der Insulin-produzierenden Betazellen verantwortlich ist. Durch die Zellzerstörung kann es zum Verlust der Produktion von Insulin und somit zu Diabetes kommen.

Das identifizierte Protein, MST1, ist in diabetischen Inseln aktiviert und führt zu deren Funktionsverlust und Absterben. Eine Hemmung von MST1 hingegen bewirkte einen Schutz der Betazellen vor der Zerstörung und eine Verbesserung des Überlebens in bereits diabetischen Betazellen. In Modellen von Typ-1- und Typ-2-Diabetes konnten die Forscher zudem zeigen, dass ein gezieltes Ausschalten von MST1 den Ausbruch der Erkrankung verhindert. Mit Hilfe dieser Erkenntnisse werden neue Therapieansätze gegen Diabetes möglich, die die Betazellen schützen und damit der Ursache von Diabetes entgegenwirken könnten.

Quelle: Universität Bremen, www.uni-bremen.de 